\title{
FITOTOXICIDADE DE FUNGICIDAS, ACARICIDAS E INSETICIDAS, SOBRE O MAMOEIRO (Carica papaya L.) CULTIVAR SUNRISE SOLO IMPROVED LINE 72/12 EM CONDIÇÕES DE CAMPO ${ }^{1}$
}

\author{
ALCÍLIO VIEIRA², CARLOS RUGGIERO³ E SÉRGIO LUCIO DAVID MARIN ${ }^{4}$
}

\begin{abstract}
RESUMO - A presente pesquisa teve como objetivo estudar os efeitos fitotóxicos de fungicidas, acaricidas e inseticidas e algumas associações entre eles, em plantas de mamoeiros (Carica papaya L.) cv. Sunrise Solo Improved Line 72/12, em condições de campo, no município de São Mateus - ES, pertencente à maior região produtora do Estado. O experimento foi arranjado em delineamento de blocos casualizados, com 4 repetições e 03 plantas úteis por parcela. Foram utilizados os seguintes produtos, com as respectivas doses, para cada 100 L de água: chlorothalonil (Daconil PM-200g); mancozeb (Dithane PM - 200g); oxicloreto de cobre (Reconil 400g); thiabendazole (Tecto 450 - 100ml); dicofol + tetradifon (Carbax - 200ml); triazophós (Hostathion $400 \mathrm{BR}-150 \mathrm{ml})$; óxido de fenbutatina (Torque $500 \mathrm{SC}-60 \mathrm{ml}$ ); e abamectin (Vertimec $18 \mathrm{CE}-50 \mathrm{ml}$ ): Analisou-se a fitotoxicidade dos produtos testados, em relação à altura da planta, $\mathrm{n}^{\circ}$ de folhas, número de flores e frutos ; diâmetro do caule e queimaduras ou injúrias foliares. As datas das avaliações foram: 01 dia antes das pulverizações, 15 dias e 30 dias após as mesmas. Os fungicidas Daconil BR, Reconil e Tecto 450; o fungicida acaricida Dithane PM; os acaricidas Carbax e Torque 500 SC; e o inseticida-acaricida Vertimec 18 CE, aplicados isoladamente, não afetaram o crescimento e a produção das plantas, nem causaram injúrias nas folhas das mesmas. A associação de fungicidas e fungicida-acaricida, com os acaricidas, ou inseticida-acaricida, não mostrou nenhum efeito fitotóxico sobre os parâmetros de crescimento avaliados, nem causaram queimaduras ou injúrias foliares.
\end{abstract}

Termos de indexação: mamão, defensivos e produtos fitossanitários.

\section{TOXIC EFFECTS OF FUNGICIDES, ACARICIDES AND INSECTICIDES, ON PAPAYA PLANT (Carica papaya L.) cv. SUNRISE SOLO IMPROVED LINE 72/12, UNDER FIELD CONDITIONS.}

\begin{abstract}
The objective of this work was to evaluate the phytotoxic effect of insecticides, acaricides and fungicides alone and in combination, on papaya plants, cv. Sunrise Solo Improved Line 72/12, under field and summer conditions. The experiment were conducted in a private farm, located at São Mateus county, the most important papaya region in the State of Espírito Santo. The experiment, arranged in a randomized block design with four replications, were conducted. Each plot consisted of three plants. The products with their respective dose in a volume of $100 \mathrm{l} \mathrm{H}_{2} \mathrm{O}$ were:chlorothalonil (Daconil PM -200g); mancozeb (Dithane PM 200g); Cupric oxychloride (Reconil - 400g); thiabendazole (Tecto 45 - 100ml).; dicofol + tetradifon (Carbax - 200ml); triazophós (Hostathion $400 \mathrm{BR}-150 \mathrm{ml}$ ); fenbutatin oxide (Torque $500 \mathrm{SC}-60 \mathrm{ml}$ ); and abamectin (Vertimec $18 \mathrm{CE}$ - 50ml). Hostathion 400 BR was phytotoxic to adult papaya plants, mainly when associated with other chemical products. The fungicides Daconil BR, Reconil and Tecto 450; the fungicide-acaricide Dithane PM; the acaricides Carbax and Torque 500 SC; and the inseticide-acaricide Vertimec 18 $\mathrm{CE}$, applied lonely, did not affect plant growth and production, and did not cause leaf and burns injury. These association of fungicides and fungicide-acaricide, with each other acaricides or inseticide-acarice, showed neither phytotoxic reactions, growth pattern alterations nor leaf injury and burning.
\end{abstract}

Index terms: papaya, phytotoxic effects, pesticide products.

\section{INTRODUÇÃO}

O mamoeiro Carica papaya L. pertence à família Caricaceae, sendo o gênero Carica o mais conhecido.

Com a ocorrência do mosaico-do-mamoeiro houve a migração da cultura, de São Paulo, principalmente para a Bahia, Espírito Santo e Pará, onde se cultivam, na grande maioria, os mamoeiros de sementes importadas, e/ou seus avanços genéticos, notadamente, a cultivar Sunrise Solo Line 72/12 (Martelleto et al., 1997).

Diversas pragas e doenças fúngicas também ocorrem na região do Norte do Espírito Santo (atualmente a mais importante no Brasil), necessitando de controle, quer nas diversas fases de desenvolvimento das plantas, quer em frutos pós-colheita (Marin et al., 1995). Para este controle, são necessárias pulverizações periódicas, o que acarreta problemas de

\footnotetext{
1 Trabalho $n^{\circ}$ 100/2001. Recebido: 09/03/2001. Aceito para publicação: 20/07/2001.

2 Pesquisador, Dr. da PESAGRO-RIO, Estação Experimental de Macaé, Estrada Velha de Glicério Km 03. CEP. 27901-970, Cx. Postal 119.371, Macaé-RJ. pesagro@castelo.com.br

3 Professor Titular de Fruticultura, Departamento de Produção Vegetal - FCAV/UNESP, Via de Acesso Prof. Paulo Donato Castellane, km 05. CEP. 14884-900 - Jaboticabal-SP. E-mail: ruggiero@fcav.unesp.br.

4 Professor Assistente, M.Sc., Centro de Ciências e Tecnologias Agropecuárias, UENF, Avenida Alberto Lamego, 2000 - Horto - CEP. 28015620, Campos-RJ.
} 
fitotoxicidade, agravados pela sensibilidade do mamoeiro à aplicação de defensivos.

Marin et al. (1995), dentre vários produtos, recomendaram, para o controle do ácaro-branco do mamoeiro, o uso do abamectin + óleo mineral (Vertimec $18 \mathrm{CE}-50 \mathrm{ml}+$ Naturoil $250 \mathrm{ml}$ ), abamectin + clofentezine (Vertimec $18 \mathrm{CE}$ $25 \mathrm{ml}+$ Acaristop $500 \mathrm{SC}-20 \mathrm{ml}$ ); para o ácaro-rajado e ácarovermelho, Vertimec $18 \mathrm{CE}$ a $50 \mathrm{ml}$, óxido de fenbutatina (Torque $500 \mathrm{SC}-80$ a $100 \mathrm{ml}$ e Partner SC - 80 a $100 \mathrm{ml}$ ) e dicofol + tetradifon (Carbax ou Acardifon - $200 \mathrm{ml}$ ), todas recomendações em 1001 de água.

Medina (1980) indicou, para o controle da varíola ou pinta-preta, o uso de fungicidas cúpricos, como Cupravit Azul e Cupravit Verde, nas dosagens de 0,30\%. Para o controle da antracnose, o mancozeb (Dithane M 45 a 0,24\%). Para o controle do oídio ou míldio, enxofre $80 \mathrm{PM}$ de 0,60 a $0,70 \%$, quinomethionate (Morestan $25 \mathrm{PM}$ a $0,05 \%$ ) e triazophós (Afugan $30 \mathrm{CE}$ a $0,08 \%$ ). Para o controle da podridão-dopedúnculo, utilizar propineb (Antracol $70 \mathrm{PM}$ a 0,25\%).

Marin et al. (1995) recomendaram o uso de diversos agrotóxicos, para o controle de doenças do mamoeiro, dentre estes, podem ser citados para o controle da antracnose, mancozeb (Dithane M 45 - 150 a $200 \mathrm{~g}$ ), chlorothalonil (Daconil $\mathrm{Br}$ $200 \mathrm{~g}$ ), thiabendazole (Tecto $600-150 \mathrm{~g}$ ), oídio, chlorothalonil (Daconil BR - $200 \mathrm{~g}$ ); podridão-do-pedúnculo do fruto e mancha de ascoquita, chlorothalonil (Daconil BR $-200 \mathrm{~g}$ ), mancozeb (Dithane M-45 - 150 a 200g), thiabendazole (Tecto $600-100$ g), todas as doses diluídas em solução contendo 100 litros de água.

Foram conduzidos dois experimentos por Marin (1988), usando-se mudas envasadas, em casa de vegetação, na FCAV UNESP, em Jaboticabal -SP, para estudar os efeitos fitotóxicos de inseticidas, acaricidas e fungicidas, no mamoeiro 'Sunrise Solo Improved Line 72/12'; algumas de suas conclusões foram: o triazophós (Hostathion $40 \mathrm{CE}$ ), causou a redução do diâmetro do caule e do número de folhas das plantas, além de apresentar extrema fitotoxicidade às folhas das plantas jovens; tetradifon + dicofol (Carbax 16 CE), mancozeb (Dithane M 45), oxicloreto de cobre (Recop 84 PM) e chlorotalonil (Daconil 75 PM) não causaram queimaduras às folhas, ou estas foram leves, aparentemente não afetando o seu desenvolvimento.

Em relação aos níveis de fitotoxicidez, Marin et al. (1995) afirmaram que o Hostathion $40 \mathrm{CE}$ ou Hostathion 400 BR, utilizados a $100 \mathrm{ml}$, causam severo desfolhamento em plantas de mamoeiro; o Carbax (240ml), Dithane M 45 (200g), Recop $(50 \% \mathrm{PM}-250 \mathrm{~g})$, Vertimec $18 \mathrm{CE}(50 \mathrm{ml})$, Torque $500 \mathrm{SC}$ (100 ml), praticamente não são tóxicos, e as folhas permanecem normais, com pouca ou quase nenhuma mancha.

Vieira et al. (1998) avaliaram a fitotoxicidade de diversos produtos, sobre mamoeiros da cv. Sunrise Solo Improved Line 72/12, em condições de campo, após a "sexagem" , em São Mateus - ES. Os produtos foram aplicados isoladamente, ou em mistura; concluíram que abamectin (Vertimec $18 \mathrm{CE}$ ) + oxicloreto de cobre (Reconil) ou thiabendazole (Tecto 450) ocasionaram leves injúrias foliares, 15 dias após as pulverizações; e que mancozeb (Dithane PM), Reconil, Tecto 450, dicofol +tetradifon (Carbax), óxido de fenbutatina (Torque $500 \mathrm{SC}$ ), Dithane PM + Vertimec $18 \mathrm{CE}$, Dhitane PM + Torque 500 SC Reconil + Carbax, Reconil +
Torque 500 SC, Tecto 450 + Carbax, e Tecto 450 + Torque 500 $\mathrm{SC}$, não interfiriram no desenvolvimento e na produção, nem causaram injúrias, ou queimaduras nas folhas.

\section{MATERIAL E MÉTODOS}

O experimento foi instalado em propriedade particular, no município de São Mateus-ES, localizado a $19^{\circ} .25^{\prime}$ de latitude sul e $40^{\circ} 03^{\prime}$ 'de longitude oeste, em solo do tipo Latossolo Vermelho Distrófico (LVD) 11, de clima típico Awi.

A formação das mudas e todo o sistema de produção utilizado foram o proposto por Marin et al. (1995).

A cultura foi irrigada por aspersão convencional e de acordo com as necessidades da mesma.

Embora não houvesse condições de aferir no local os dados necessários, na prática, observou-se que as condições, durante o desenvolvimento dos trabalhos, foram semelhantes às condições de São Mateus, que apresentava, nos meses estudados, precipitações mais elevadas e temperaturas mais altas do que nos períodos "mais frescos" do ano.

O delineamento experimental foi em blocos casualizados, com 25 tratamentos, 04 repetições e 3 plantas úteis por parcela experimental.

As avaliações foram efetuadas um dia antes das pulverizações, 15 dias e 30 dias após.

Os dados relativos aos efeitos fitotóxicos foram analisados estatisticamente, aplicando-se o teste $\mathrm{F}$, e as médias comparadas através do teste de Tukey, a $5 \%$ de probabilidade.

\begin{tabular}{llll}
\hline \multicolumn{2}{c}{ Os tratamentos utilizados foram: } & $\begin{array}{c}\text { Dosagem do produto } \\
\text { Produtos }\end{array}$ & $\begin{array}{c}\text { Nomercial para } 100 \\
\text { litros água }\end{array}$ \\
\hline Trat. & Nome Comercial & chlorothalonil & $200 \mathrm{~g}$ \\
\hline 1 & Daconil & mancozeb & $200 \mathrm{~g}$ \\
2 & Dithane PM & oxicloreto de cobre & $400 \mathrm{~g}$ \\
3 & Reconil & thiabendazole & $100 \mathrm{ml}$ \\
4 & Tecto 450 & dicofol+tetradifon & $200 \mathrm{ml}$ \\
5 & Carbax & triazophós & $150 \mathrm{ml}$ \\
6 & Hostathion 400 BR & óxido de fenbutatina & $60 \mathrm{ml}$ \\
7 & Torque 500 Sc & abamectin & $50 \mathrm{ml}$ \\
8 & Vertimec 18 CE & chlorothalonil+(dicofol+tetradifon) & $200 \mathrm{~g}+200 \mathrm{ml}$ \\
9 & Daconil BR + Carbax & chlorothalonil+triazophós & $200 \mathrm{~g}+150 \mathrm{ml}$ \\
10 & Daconil BR+Hostathion 400 BR & $200 \mathrm{~g}+60 \mathrm{ml}$ \\
11 & Daconil BR+Torque 500 SC & chlorothalonil+óx.fenbutatina & $200 \mathrm{~g}+50 \mathrm{ml}$ \\
12 & Daconil BR+Vertimec 18 CE & chlorothalonil+abamectin & $200 \mathrm{~g}+200 \mathrm{ml}$ \\
13 & Dithane PM+Carbax & mancozeb+(dicofol+tetradifon) & $200 \mathrm{~g}+150 \mathrm{ml}$ \\
14 & Dithane PM+Hostathion 400 BR & mancozeb+triazophós & $200 \mathrm{~g}+60 \mathrm{ml}$ \\
15 & Dithane PM+Torque 500 SC & mancozeb+óxido de fenbutatina & $200 \mathrm{~g}+50 \mathrm{ml}$ \\
16 & Dithane PM+Vertimec 18 CE & mancozeb+abamectin & $400 \mathrm{~g}+200 \mathrm{ml}$ \\
17 & Reconil+Carbax & óx.cobre+(dicofol+tetradifon) & $400 \mathrm{~g}+150 \mathrm{ml}$ \\
18 & Reconil+Hostathion 400 BR & óx.cobre+triazophós & $400 \mathrm{~g}+60 \mathrm{ml}$ \\
19 & Reconil+Torque 500 SC & óx.cobre+óxidode fenbutatina & $400 \mathrm{~g}+50 \mathrm{ml}$ \\
20 & Reconil+Vertimec 18 CE & óx.cobre+abamectin & $100 \mathrm{ml}+200 \mathrm{ml}$ \\
21 & Tecto 450+Carbax & thiabendazole+(dicofol+tetradifon) & $100 \mathrm{ml}+150 \mathrm{ml}$ \\
22 & Tecto 450+Hostathion 400 BR & thiabendazole+triazophós & $100 \mathrm{ml}+60 \mathrm{ml}$ \\
23 & Tecto 450+Torque 500 SC & thiabendazole+óxido de fenbutatina & $100 \mathrm{ml}+50 \mathrm{ml}$ \\
24 & Tecto 450+Vertimec 18 CE & thiabendazole+abamectin & 100 \\
25 & Testemunha (água) & & \\
\hline & & &
\end{tabular}

Avaliaram-se os parâmetros: de crescimento da planta - altura da planta, diâmetro do caule (a $10 \mathrm{~cm}$ do solo) e número de folhas relacionadas à produção - número de flores e frutos.

Os sintomas visuais de fitotoxicidade (injúrias e/ou queimaduras nas folhas) foram verificados por 02 observadores, através da seguinte escala visual de notas, utilizada por Marin (1988):

Nota 0 - plantas com folhas normais, sem sintomas de queimaduras;

Nota 1 - plantas com folhas levemente injuriadas e/ou com 
pequenas áreas queimadas;

Nota 2 - plantas com folhas medianamente injuriadas, amareladas e com bordas e ápices queimados;

Nota 3 - plantas com folhas intensamente injuriadas, apresentando severo desfolhamento.

\section{RESULTADOS E DISCUSSÃO}

Ao fazer as avaliações da intensidade de queimadura nas plantas estudadas, constatou-se que somente os tratamentos, onde constava a aplicação do Hostathion (Tabela 1), ocasionaram queimaduras e injúrias nas folhas dos mamoeiros.
Todos os outros produtos, aplicados isoladamente ou associados, não causaram nenhum dano às folhas dos mamoeiros.

No trabalho desenvolvido por Marin (1988), o triazophós (Hostathion $40 \mathrm{CE}$ ) também causou fitotoxicidade às folhas das plantas jovens. Afirmou que o Carbax $16 \mathrm{CE}$, Dithane M 45, oxicloreto de cobre (Recop 84 PM) e Daconil 75 PM podem causar injúrias às folhas destas plantas, o que não se verificou no presente trabalho, porque, provavelmente, é maior a resistência à fitotoxicidade de plantas mais adultas (no campo, após sexagem) e em pleno desenvolvimento, favorecidas pôr condições propícias ao crescimento dos mamoeiros.

Em condições climáticas mais amenas, Vieira et al. (1998) constataram, no mesmo local do presente trabalho, que o Vertimec $18 \mathrm{CE}$, associado ao Recomil ou ao Tecto 450, pode

TABELA 1 - Efeito da aplicação do Hostathion 400 BR sobre a intensidade de queimadura em folhas de mamoeiros, cv. Sunrise Solo Improved Line 72/12, em condições de campo (São Mateus-ES).

\begin{tabular}{|c|c|c|}
\hline TR A T A M ENTOS & \multicolumn{2}{|c|}{ Intensidade de queim aduras ${ }^{1}$} \\
\hline Nomes Comerciais & $\begin{array}{l}15 \text { dias após } \\
\text { as aplicações }\end{array}$ & $\begin{array}{c}30 \text { dias após as } \\
\text { aplicações }\end{array}$ \\
\hline Hostathion $400 \mathrm{BR}$ & 2,00 & 0,50 \\
\hline Hostathion $400 \mathrm{BR}+\mathrm{Daconil} \mathrm{B} R$ & 2,00 & 1,00 \\
\hline Hostathion $400 \mathrm{BR}+$ Dithane PM & 2,00 & 0,80 \\
\hline Hostathion $400 \mathrm{BR}+\mathrm{R}$ econil & 2,00 & 0,25 \\
\hline Hostathion $400 \mathrm{BR}+\mathrm{T}$ ecto 450 & 2,00 & 0,90 \\
\hline
\end{tabular}

1 Média de notas atribuídas por dois observadores. Escala de notas: $0=$ sem injúrias, 3 = injúria máxima.

TABELA 2 - Influência das aplicações de fungicidas, acaricidas e inseticidas sobre a altura de plantas e diâmetro de caules de mamoeiros, cv. Sunrise Solo Improved Line 72/12, em condições de campo.

\begin{tabular}{|c|c|c|c|c|c|c|}
\hline Tratamentos & \multicolumn{3}{|c|}{ A ltura das plantas(cm) } & \multicolumn{3}{|c|}{ D iâmetro do caule(cm) } \\
\hline Nomes comerciais & 1 & 2 & 3 & 1 & 2 & 3 \\
\hline 1. Daconil B R & 144,16 & $161,50 \mathrm{ab}$ & 193,96 & 4,75 & 5,86 & $7,56 \mathrm{abc}$ \\
\hline 3. Reconil & 140,12 & $156,66 \mathrm{ab}$ & 191,04 & 4,69 & 5,67 & $7,46 \mathrm{abc}$ \\
\hline 4. Tecto 450 & 146,12 & $163,08 \mathrm{ab}$ & 196,96 & 5,15 & 6,15 & $8,01 \mathrm{ab}$ \\
\hline 5. C arbax & 144,37 & $160,71 \mathrm{ab}$ & 191,71 & 4,93 & 5,78 & $7,68 \mathrm{abc}$ \\
\hline 9. Daconil B R + Carbax & 153,62 & $174,21 \mathrm{ab}$ & 207,41 & 5,24 & 6,39 & $8,25 \mathrm{a}$ \\
\hline 10. Daconil B R + Hostathion 400 B R & 153,08 & $157,23 \mathrm{ab}$ & 180,75 & 5,05 & 5,70 & $6,01 \mathrm{~cd}$ \\
\hline 11. Daconil B R + Torque $500 \mathrm{SC}$ & 144,58 & $163,70 \mathrm{ab}$ & 194,95 & 5,05 & 6,11 & $7,78 \mathrm{abc}$ \\
\hline 12. Daconil B R + Vertimec CE & 139,79 & $156,83 \mathrm{ab}$ & 190,58 & 4,78 & 5,71 & $7,37 \mathrm{abc}$ \\
\hline 13. Dithane $P M+C$ arbax & 137,66 & $151,04 \mathrm{ab}$ & 181,91 & 4,59 & 5,40 & $6,798 \mathrm{abcd}$ \\
\hline 14. Dithane PM + Hostathion 400 B R & 151,67 & $159,35 \mathrm{ab}$ & 182,88 & 5,00 & 5,32 & $6,06 \mathrm{~cd}$ \\
\hline 20. Reconil + Vertimec $18 \mathrm{CE}$ & 142,08 & $160,12 \mathrm{ab}$ & 195,41 & 4,59 & 5,42 & $7,14 \mathrm{abcd}$ \\
\hline 21. Tecto $450+\mathrm{Carbax}$ & 154,87 & $174,79 \mathrm{a}$ & 206,37 & 5,13 & 6,32 & $8,13 \mathrm{a}$ \\
\hline 22. Tecto $450+$ Hostathion 400 B R & 138,83 & $143,87 \mathrm{~b}$ & 163,16 & 4,37 & 4,83 & $5,23 \mathrm{~d}$ \\
\hline 23. Tecto $450+$ Torque $500 \mathrm{SC}$ & 143,16 & $162,62 \mathrm{ab}$ & 198,46 & 4,75 & 5,70 & $7,57 \mathrm{abc}$ \\
\hline 24. Tecto + Vertimec $18 \mathrm{CE}$ & 141,33 & $157,37 \mathrm{ab}$ & 187,37 & 4,71 & 5,54 & $7,24 a b c$ \\
\hline 25. Testemunha (água) & 151,25 & $168,75 \mathrm{ab}$ & 204,12 & 4,68 & 5,80 & $7,38 \mathrm{abc}$ \\
\hline F (Tratamentos) & $1,11 \mathrm{~N} \mathrm{~S}$ & $1,83^{*}$ & $1,12 \mathrm{~N} \mathrm{~S}$ & $0,57 \mathrm{~N} \mathrm{~S}$ & $1,47 \mathrm{~N} \mathrm{~S}$ & $4,64 * *$ \\
\hline D.M.S. & - & 30,41 & - & - & - & 1,93 \\
\hline $\mathrm{C} V(\%)$ & 22,57 & 7,07 & 23,97 & 12,03 & 12,37 & 10,82 \\
\hline
\end{tabular}

$\mathrm{NS}=$ Não Significativo.**Significativo ao nível de1\% de probabilidade. ${ }^{*}$ Significativo ao nível de $5 \%$ de probabilidade. Médias seguidas de, pelo menos, uma letra igual não diferem entre si, pelo teste de Tukey, ao nível de $5 \%$ de probabilidade.

Datas das contagens: $1=01$ dia antes das pulverizações; $2=15$ dias após as pulverizações; e $3=30$ dias após as pulverizações. 
TABELA 3 - Influência das aplicações de fungicidas, acaricidas e inseticidas sobre o número de folhas e de flores e frutos de mamoeiros, cv. Sunrise Solo Improved Line 72/12, em condições de campo (São Mateus-ES).

\begin{tabular}{|c|c|c|c|c|c|c|}
\hline \multirow[t]{2}{*}{ Tratamentos } & \multicolumn{3}{|c|}{$\mathrm{N}^{\circ}$ de folhas } & \multicolumn{3}{|c|}{$\mathrm{N}^{\circ}$ de flores e frutos } \\
\hline & \multicolumn{3}{|c|}{ Datas das contagens } & \multicolumn{3}{|c|}{ Datas das contagens } \\
\hline Nomescomerciais & 1 & 2 & 3 & 1 & 2 & 3 \\
\hline 1. D a conil B R & 22,66 & $26,08 \mathrm{ab}$ & $33,50 \mathrm{a}$ & 16,16 & $21,41 \mathrm{abcd}$ & $85,08 \mathrm{ab}$ \\
\hline 3. Reconil & 23,33 & $26,50 \mathrm{ab}$ & $32,41 \mathrm{a}$ & 15,75 & $23,25 \mathrm{abcd}$ & $76,58 \mathrm{ab}$ \\
\hline 4. Tecto 450 & 24,49 & $27,92 \mathrm{ab}$ & $33,33 \mathrm{a}$ & 18,25 & $29,25 \mathrm{ab}$ & $95,75 \mathrm{a}$ \\
\hline 5. C arbax & 23,08 & $27,08 \mathrm{ab}$ & $31,67 \mathrm{a}$ & 16,92 & $25,08 \mathrm{abcd}$ & $78,50 \mathrm{ab}$ \\
\hline 9. Daconil B R + Carbax & 25,00 & $29,91 \mathrm{a}$ & $33,25 \mathrm{a}$ & 21,33 & $32,00 \mathrm{a}$ & $91,25 \mathrm{a}$ \\
\hline 10.Daconil B R + Hostathion 400 B R & 24,62 & $22,92 \mathrm{ab}$ & $17,54 \mathrm{~b}$ & 18,54 & $10,46 \mathrm{~d}$ & $19,75 \mathrm{e}$ \\
\hline 11.Daconil B R + Torque $500 \mathrm{SC}$ & 23,58 & $29,08 \mathrm{a}$ & $33,25 \mathrm{a}$ & 17,66 & $30,00 \mathrm{ab}$ & $87,83 \mathrm{a}$ \\
\hline 12.Daconil B R + Vertimec CE & 22,17 & $27,25 \mathrm{ab}$ & $32,66 \mathrm{a}$ & 15,17 & $27,08 \mathrm{abcd}$ & $79,75 \mathrm{ab}$ \\
\hline 13. Dithane PM + Carbax & 21,25 & $25,00 \mathrm{ab}$ & $30,58 \mathrm{a}$ & 14,75 & $18,76 \mathrm{abcd}$ & $61,25 \mathrm{abcde}$ \\
\hline 14. Dithane PM + Hostathion 400 B R & 24,33 & $23,75 \mathrm{ab}$ & $20,17 \mathrm{~b}$ & 17,91 & $11,33 \mathrm{~cd}$ & $28,00 \mathrm{de}$ \\
\hline 15. Dithane PM + Torque 500 SC & 21,00 & $25,33 \mathrm{ab}$ & $29,75 \mathrm{a}$ & 15,25 & $18,83 \mathrm{abcd}$ & $60,24 \mathrm{abcde}$ \\
\hline $20 . \mathrm{Reconil}+$ Vertimec $18 \mathrm{CE}$ & 21,66 & $26,17 \mathrm{ab}$ & $31,92 \mathrm{a}$ & 14,91 & $20,75 \mathrm{abcd}$ & $73,42 \mathrm{abcd}$ \\
\hline 21. Tecto $450+\mathrm{Carbax}$ & 25,00 & $30,08 \mathrm{a}$ & $34,25 \mathrm{a}$ & 20,75 & $28,83 \mathrm{abc}$ & $90,25 \mathrm{a}$ \\
\hline 22. Tecto $450+$ Hostathion 400 B R & 22,00 & $20,91 \mathrm{~b}$ & $19,83 \mathrm{~b}$ & 14,92 & $9,91 \mathrm{~d}$ & $22,33 \mathrm{e}$ \\
\hline 23. Tecto $450+$ Torque $500 \mathrm{SC}$ & 22,83 & $27,83 \mathrm{ab}$ & $32,91 \mathrm{a}$ & 15,58 & $23,41 \mathrm{abcd}$ & $80,17 \mathrm{ab}$ \\
\hline 24. Tecto + Vertimec 18 C E & 22,41 & $26,92 \mathrm{ab}$ & $31,08 \mathrm{a}$ & 14,17 & $23,75 \mathrm{abcd}$ & $71,83 \mathrm{abcd}$ \\
\hline 25. Testemunha (água) & 22,58 & $26,66 \mathrm{ab}$ & $31,42 \mathrm{a}$ & 13,83 & $24,16 \mathrm{abcd}$ & $65,25 \mathrm{abcde}$ \\
\hline F (Tratamentos & $1,03 \mathrm{~N} \mathrm{~S}$ & $2,88 * *$ & $17,89 * *$ & $0,71 \mathrm{~N} \mathrm{~S}$ & $4,26 * *$ & $7,13 * *$ \\
\hline D.M.S. & - & 7,42 & 6,57 & - & 17,55 & 45,86 \\
\hline C V $(\%)$ & 10,21 & 10,45 & 8,14 & 29,42 & 29,02 & 24,63 \\
\hline
\end{tabular}

ao nível de $1 \%$ de probabilidade.Contagens: $1=01$ dia antes das pulverizações; $2=15$ dias após as pulverizações; e $3=30$ dias após as pulverizações.

ocasionar leves injúrias às folhas dos mamoeiros; estes efeitos não foram encontrados na presente pesquisa. Os demais tratamentos testados por esses autores, também naquele experimento, não causaram nenhuma injúria ou queimadura foliar.

Pode-se constatar, pelos resultados apresentados na Tabela 2, que somente a associação entre o Hostathion 400 BR e o Tecto 450 afetou negativamente o crescimento das plantas, em relação à altura das mesmas. Os efeitos dos produtos, sobre o diâmetro dos caules, também evidenciam o efeito fitotóxico do Hostathion $400 \mathrm{BR}$, principalmente associado às misturas com os fungicidas testados: Daconil BR, Dithane PM, Reconil e Tecto 450. Efeitos semelhantes foram obtidos por Marin (1988), em relação ao Hostathion 400 BR. Ao comparar-se os efeitos dos outros produtos, aplicados isoladamente ou em associação, estes não afetaram o desenvolvimento em altura e diâmetro das plantas, coincidindo com os resultados do trabalho desenvolvido por Vieira et al. (1998).

Os resultados advindos das contagens do número de flores e frutos (Tabela 3) nos conduzem, também, à constatação do efeito altamente fitotóxico do Hostathion 400 BR, aplicado isoladamente ou associado aos fungicidas testados.. Vieira et al. (1998) também não constataram redução do número de flores e frutos em relação aos efeitos do Dithane PM, Reconil, Tecto 450 (fungicidas), Carbax, Torque $500 \mathrm{SC}$ e Vertimec $18 \mathrm{CE}$ (acaricidas, inseticida-acaricida), aplicados isoladamente ou quando da associação entre fungicidas, com acaricidas, e inseticida-acaricida.

\section{CONCLUSÕES}

Com base nos resultados obtidos, nas condições em que foi realizado o presente trabalho, pode-se concluir que:

1. O Hostathion $400 \mathrm{BR}$ apresentou fitotoxicidade em mamoeiros adultos, principalmente quando associado aos outros produtos, ou seja, Daconil BR, Dithane PM, Reconil e Tecto 450 .

2. Os outros produtos, aplicados isoladamente ou associados, não afetaram os parâmetros de crescimento e produção das plantas, também não ocasionaram queimaduras ou injúrias foliares.

3. Deve-se considerar, sempre, ao fazer-se avaliações de fitotoxicidade de produtos fitossanitários em mamoeiros, o estágio de desenvolvimento das plantas, o balanço hídricoclimático e as normais de temperatura e umidade relativa do ar, do local da experimentação, por ocasião da pesquisa.

\section{REFERÊNCIAS BIBLIOBRÁFICAS}

AGRIANUAL. 1995: Anuário da agricultura brasileira. São Paulo: FNP Consultoria \& Comércio, 1996. p.268-72

MARIN, S.L.D. Efeitos fitotóxicos de inseticidas, acaricidas e fungicidas em mudas de mamoeiro (Carica papaya $\mathrm{L}$.) $\mathrm{cv}$. Solo. 1988. 97p. Dissertação (Mestrado em Produção Vegetal) 
- Faculdade de Ciências Agrárias e Veterinárias, Universidade Estadual Paulista, Jaboticabal, 1988.

MARIN, S.L.D, et al. Recomendações para a cultura do mamoeiro dos grupos Solo e Formosa no Estado do Espírito Santo. 4.ed. Vitória : EMCAPA, 1995. 57p. (Circular Técnica, 3).

MARTELLETO, L. A.P., MALDONADO, J.F.M., VIEIRA, A, et al. A cultura do mamão: perspectivas, tecnologias e viabilidade. Niterói: PESAGRO-RIO, 1997. 28p. (Documentos, 37).
MEDINA, J.C. Mamão - cultura. In: INSTITUTO DE TECNOLOGIA DE ALIMENTOS. Mamão da cultura ao processamento e comercialização. Campinas : ITAL, 1980. P. 7-112. (Frutas Tropicais, 7).

VIEIRA, A, RUGGIERO, C. MARIN, S.L.D. Fitotoxicidade de produtos de uso fitossanitário sobre o mamoeiro (Carica papaya L). Cultivar Sunrise Solo Improved Line 72/12. In: CONGRESSO BRASILEIRO DE FRUTICULTURA, 15., 1998, Poços de Caldas. Resumos... Lavras : UFLA, 1998. p. 486. 